MATHEMATICS OF COMPUTATION

Volume 67, Number 223, July 1998, Pages 1247-1252

S 0025-5718(98)00962-4

\title{
REMARKS ON THE SCHOOF-ELKIES-ATKIN ALGORITHM
}

\author{
L. DEWAGHE
}

\begin{abstract}
Schoof's algorithm computes the number $m$ of points on an elliptic curve $E$ defined over a finite field $\mathbb{F}_{q}$. Schoof determines $m$ modulo small primes $\ell$ using the characteristic equation of the Frobenius of $E$ and polynomials of degree $O\left(\ell^{2}\right)$. With the works of Elkies and Atkin, we have just to compute, when $\ell$ is a "good" prime, an eigenvalue of the Frobenius using polynomials of degree $O(\ell)$. In this article, we compute the complexity of Müller's algorithm, which is the best known method for determining one eigenvalue and we improve the final step in some cases. Finally, when $\ell$ is "bad", we describe how to have polynomials of small degree and how to perform computations, in Schoof's algorithm, on $x$-values only.
\end{abstract}

\section{INTRODUCTION}

Let $E$ be an elliptic curve defined over the finite field $\mathbb{F}_{q}$ of large characteristic $p$. The set of $\mathbb{F}_{q}$-points of $E$, denoted $E\left(\mathbb{F}_{q}\right)$, is a finite abelian group [20].

In 1985, Schoof [17] gave a deterministic polynomial-time algorithm for computing $\# E\left(\mathbb{F}_{q}\right)$. The algorithm determines the characteristic equation of the Frobenius $\pi$ of $E$, acting on the $\ell$-torsion points $E[\ell]$ of $E$, for $\ell$ prime. But, working on $E[\ell]$ uses computations on polynomials modulo the $\ell$-th division polynomial $f_{\ell}$, and this is not practical, due to the size of $f_{\ell}$.

In 1991, Elkies [10] showed how to perform computations in the kernel of an isogeny of degree $\ell$, by computing a factor of degree $d=(\ell-1) / 2$ of $f_{\ell}$. This idea works for nearly half the primes $\ell$, called Elkies primes. For such an $\ell$, the algorithm has just to compute an eigenvalue of $\pi$ acting on $E[\ell]$.

Atkin [1] had given in 1988 the sort and match method used now for "bad" primes $\ell$. Then he made the algorithm practical for very large finite fields [2] and the method became the SEA (for Schoof-Elkies-Atkin) algorithm.

For the last improvements in this scope, see [5], [6] and [12] and for the case $p$ small, see [7] and the implementation in [13].

In this article we compute, for an Elkies prime $\ell$, the complexity of the best asymptotic method used for computing an eigenvalue of $\pi$ over $E[\ell]$ and we show then how to avoid, in some cases, the computation with $y$-coordinates of points. Finally, for a bad prime $\ell$, we explain how to obtain a proper factor of $f_{\ell}$ and show then how to avoid again, in Schoof's algorithm, computations with $y$-coordinates of points.

Received by the editor May 11, 1996 and, in revised form, October 2, 1996 and February 19, 1997.

1991 Mathematics Subject Classification. Primary 14H52, 14K02, 11 Y16.

Key words and phrases. Elliptic curves, finite fields, Schoof algorithm, division polynomials, computational number theory. 
These results have enabled Morain [15] to compute $\# E\left(\mathbb{F}_{p}\right)$, for $p$ prime of 500 digits (this is the actual record).

\section{The SEA ALGORITHM}

2.1. Elliptic curve over $\mathbb{F}_{q}$. Let $E$ be a non-supersingular elliptic curve given by an affine equation $\mathcal{F}(x, y)=0$ where

$$
\mathcal{F}(x, y)=y^{2}+a_{1} x y+a_{3} y-\left(x^{3}+a_{2} x^{2}+a_{4} x+a_{6}\right)
$$

with the $a_{i}$ 's in $\mathbb{F}_{q}$.

The set $E\left(\mathbb{F}_{q}\right)=\left\{(x, y) \in \mathbb{F}_{q} \times \mathbb{F}_{q}, \mathcal{F}(x, y)=0\right\} \cup\left\{O_{E}\right\}$ is an abelian group and the law, denoted $\oplus$, has $O_{E}=[0: 1: 0]$ as neutral element. We denote by $f_{n}$ the $n$-th division polynomial in $x$. The degree of $f_{n}$ is $\left(n^{2}-1\right) / 2$ if $n$ is odd. The group of $n$-torsion points, $E[n]=\left\{P \in E\left(\overline{\mathbb{F}}_{q}\right) \mid n P=O_{E}\right\}$ can be represented by $\mathbb{F}_{q}[x, y] /\left(f_{n}(x), \mathcal{F}(x, y)\right)$ (see $\left.[18]\right)$.

The morphism $\pi: E\left(\overline{\mathbb{F}}_{q}\right) \rightarrow E\left(\overline{\mathbb{F}}_{q}\right),(x, y) \mapsto\left(x^{q}, y^{q}\right)$ of $E$ satisfies $\pi^{2}-t \pi+q=0$ over $E\left(\overline{\mathbb{F}}_{q}\right)$, with $t \in \mathbb{Z}$, satisfying $|t| \leq 2 \sqrt{q}$. Recall : $\# E\left(\mathbb{F}_{q}\right)=q+1-t$. When $\ell$ is an odd prime number (see [6] for $\ell=2$ ), we consider the restriction $\pi_{\ell}$ of $\pi$ to $E[\ell]$, which satisfies $\pi_{\ell}^{2}-\tau \pi_{\ell}+k=0$ over $E[\ell]$ with $t \equiv \tau \bmod \ell$ and $q \equiv k \bmod \ell$. Now, if $\ell \neq p, E[\ell] \cong \mathbb{Z} / \ell \mathbb{Z} \times \mathbb{Z} / \ell \mathbb{Z}$, so we can view $E[\ell]$ as a vector space over $\mathbb{F}_{\ell}$ and $x^{2}-\tau x+k$ as the characteristic equation of $\pi_{\ell}$. We denote by $G_{1}, G_{2}, \ldots, G_{\ell+1}$ the $(\ell+1)$ cyclic subgroups of $E\left(\overline{\mathbb{F}}_{q}\right)$, of order $\ell$.

2.2. The SEA algorithm. Schoof [17] determines $\# E\left(\mathbb{F}_{q}\right)=q+1-t$ by searching for a match among the $\ell$ equations $\left(x^{q^{2}}, y^{q^{2}}\right) \oplus k(x, y)=\tilde{\tau}\left(x^{q}, y^{q}\right), 0 \leq \tilde{\tau} \leq \ell-1$, over $E[\ell]$.

Elkies works in the kernel $G_{i}$ of one of the $\ell+1$ isogenies $E \stackrel{\varrho_{i}}{\rightarrow} E_{i}, 1 \leq i \leq \ell+1$, of degree $\ell$. When $D=\tau^{2}-4 k$ is a square modulo $\ell$ the eigenvalues of $\pi_{\ell}$ are in $\mathbb{F}_{\ell}$ and $\ell$ is called an Elkies prime. Hence, in this case, the eigenspaces are $\mathbb{F}_{q^{-}}$ rational and the corresponding isogenies are defined over $\mathbb{F}_{q}$ and if we let $E[\ell]_{\lambda}$ be an eigenspace with $P_{\lambda}$ a generator, we have $h_{\ell}(x)=\prod_{i=1}^{d}\left(x-x\left(i P_{\lambda}\right)\right) \in \mathbb{F}_{q}[x]$ and

$$
E[\ell]_{\lambda}=\left\langle P_{\lambda}\right\rangle=\mathbb{F}_{q}[x, y] /\left(h_{\ell}(x), \mathcal{F}(x, y)\right) .
$$

Let $\Phi_{\ell}(x, y)=0$ be the canonical equation of the modular curve $X_{0}(\ell)$ (see [2], [15] for a simpler equation). We know that $\ell$ is an Elkies prime if and only if $\Phi_{\ell}(j(E), x)=0$ has a root in $\mathbb{F}_{q}$.

For $p \neq 2,3$ and $\ell$ an Elkies prime, the formulas of Atkin [2],[15] give, from a root of $\Phi_{\ell}(j(E), x)=0$ in $\mathbb{F}_{q}$, the value of $p_{1}=\sum_{i=1}^{d} x\left(i P_{\lambda}\right)$ and the coefficients of the corresponding $E_{i}$. So [10], one can compute the $p_{k}=\sum_{i=1}^{d} x^{k}\left(i P_{\lambda}\right)$ for $1 \leq k \leq d$ and hence $h_{\ell}$ by Newton's formula if $\ell \ll p$.

If $p=2$ or 3 or $\ell \approx p$ see Couveignes' work [7] and also [13].

Once $h_{\ell}$ is known, we have to search a match among the $\ell-1$ equations $\left(x^{q}, y^{q}\right)=$ $\tilde{\lambda}(x, y), 1 \leq \tilde{\lambda} \leq \ell-1$, over $E[\ell]_{\lambda}$.

If $D$ is not a square modulo $\ell$, then $\ell$ is called an Atkin prime and the $G_{i}$ 's are

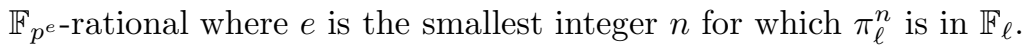

\section{LOOKING FOR ONE EIGENVALUE}

3.1. Computing $\lambda \bmod \ell$. We compute the complexity of the algorithm of Müller [16] which computes $\lambda \bmod \ell$. Müller uses an integer $k_{\text {opt }} \approx\lceil\sqrt{d}\rceil$ such that for all 
$\lambda$ in $\mathbb{F}_{\ell}^{*}$, there are integers $i, j$ with $1 \leq i, j \leq k_{\text {opt }}$ such that $\lambda \equiv \pm i / j \bmod \ell$. So, he compares $j\left(x^{q}, y^{q}\right)$ and $i(x, y)$ using division polynomials, which means comparisons of rational functions.

The elementary operation is taken to be the cost of one multiplication of two elements in $\mathbb{F}_{q}$. Let $M(d)$ be the number of operations needed to compute the multiplication of two polynomials of degree $d$ (see [11]).

Proposition 1. Müller's method takes $O(M(d) \log q)+O(\sqrt{d} M(d))+O\left(d^{2}\right)$ operations and $O(d \sqrt{d})$ space.

Proof. The computation of $x^{q} \bmod h_{\ell}(x)$ requires $O(M(d) \log q)$ operations and the computation of the $k_{\text {opt }}$ first division polynomials requires $O(\sqrt{d} M(d))$ operations.

The $x\left(j\left(x^{q}, y^{q}\right)\right)$ are computed using the recursive formulae of division polynomials in $x^{q}$ (see [8]). This requires $O(\sqrt{d} M(d))$ operations, which is more efficient than modular compositions $x(j(x, y)) \circ x^{q}$ (see [4], [19]).

To compare two rational functions modulo $h_{\ell}(x)$ in $\mathbb{F}_{q}[x]$, one can test the match using a random linear map [16] and then verify polynomial equality. So, the comparisons of coordinates takes $O(2 M(d))+O\left(2 d^{2}\right)$ operations.

3.2. The sign of $\lambda \bmod \ell$. Suppose we have integers $i, j$ such that $j \pi_{\ell}= \pm i$ over $E[\ell]_{\lambda}$, where $\ell$ is an Elkies prime. We have $\lambda \equiv \pm \lambda_{0} \bmod \ell$ with $\lambda_{0} \equiv i j^{-1} \bmod \ell$. For $\mu \in \mathbb{F}_{\ell^{2}}^{*}$, we call semi-order of $\mu$, denoted $s(\mu)$, the order of $\mu$ in $\mathbb{F}_{\ell^{2}}^{*} /( \pm 1)$.

- If $p \neq 2, E$ has an equation of the form $y^{2}=\mathcal{G}(x):=x^{3}+a_{2} x^{2}+a_{4} x+a_{6}$.

Theorem 1. Let $h_{\ell}$ be the factor of $f_{\ell}$ corresponding to $\lambda$ and $g_{\ell}$ be a factor of degree $s\left(\lambda_{0}\right)$ of $h_{\ell}$ and let $r$ be $\operatorname{Resultant}\left(g_{\ell}, \mathcal{G}\right)$. Then $\lambda=\lambda_{0}^{s\left(\lambda_{0}\right)}\left(\frac{r}{q}\right) \lambda_{0}$. When $\ell \equiv 3 \bmod 4$, one can take $g_{\ell}=h_{\ell}$.

Proof. For $s\left(\lambda_{0}\right)$ odd, we have $\pi_{\ell}^{s\left(\lambda_{0}\right)}= \pm \lambda_{0}^{s\left(\lambda_{0}\right)} I d$ over $E[\ell]_{\lambda}$. If $\pi_{\ell}^{s\left(\lambda_{0}\right)}=I d$, then $E[\ell]_{\lambda} \subset E\left(\mathbb{F}_{q^{s\left(\lambda_{0}\right)}}\right)$; hence, for all $P$ in $E[\ell]_{\lambda}, \mathcal{G}(x(P))$ is a square in $\mathbb{F}_{q^{s\left(\lambda_{0}\right)}}$, and since $\prod_{i=1}^{s\left(\lambda_{0}\right)}\left(\mathcal{G}\left(x_{i}\right)\right)=r$, with $x_{i}$ the roots of $g_{\ell}, r$ is a square in $\mathbb{F}_{q}$. Whereas, if $\pi_{\ell}^{s\left(\lambda_{0}\right)}=-I d$ over $E[\ell]_{\lambda}$, then $\left(\frac{r}{q}\right)=-1$.

Note that, if $\ell \equiv 1 \bmod 4$, then one can compute $\lambda_{0}$ using $h_{\ell}$ and then determine $\lambda$, if $s\left(\lambda_{0}\right)=s\left( \pm \lambda_{0}\right)$ is odd, using a factor of $h_{\ell}$.

- If $p=2$, let $y^{2}+x y=x^{3}+B$ (with $B \in \mathbb{F}_{2^{m}}$ ) be an equation of $E$ (see [14]).

Proposition 2. Let $h_{\ell}$ be a factor of $f_{\ell}$ corresponding to $\lambda= \pm \lambda_{0}$. If $h_{\ell}$ has a factor $g_{\ell}=x^{s\left(\lambda_{0}\right)}-\tilde{s}_{1} x^{s\left(\lambda_{0}\right)-1}+\cdots+(-1)^{s\left(\lambda_{0}\right)} \tilde{s}_{s\left(\lambda_{0}\right)}$ of odd degree $s\left(\lambda_{0}\right)$, then

$$
\lambda=\left\{\begin{array}{cl}
\lambda_{0}^{s\left(\lambda_{0}\right)} \lambda_{0} & \text { if } \operatorname{Tr}\left(\tilde{s}_{1}+B\left(\tilde{s}_{s\left(\lambda_{0}\right)-1}^{2}-2 \tilde{s}_{s\left(\lambda_{0}\right)} \tilde{s}_{s\left(\lambda_{0}\right)-2}\right) / \tilde{s}_{s\left(\lambda_{0}\right)}^{2}\right)=0, \\
-\lambda_{0}^{s\left(\lambda_{0}\right)} \lambda_{0}, & \text { otherwise. }
\end{array}\right.
$$

When $\ell \equiv 3 \bmod 4$, one can take $g_{\ell}=h_{\ell}$.

Proof. The equation $X^{2}+X=\gamma$ has a root in an extension $\mathbb{F}_{2^{n}}$ if and only if $\operatorname{Tr}(\gamma)=0$ (see [9]). Hence the points of $E[\ell]_{\lambda}=\langle P=(x, y)\rangle$ are in $\mathbb{F}_{q^{s\left(\lambda_{0}\right)}}$ if and only if $\operatorname{Tr}\left(\gamma_{i}\right)=0$, where $x_{i}=x(i P)$ and $\gamma_{i}=x_{i}+B / x_{i}^{2}$. Finally, computing $\sum_{i=1}^{s\left(\lambda_{0}\right)} \operatorname{Tr}\left(\gamma_{i}\right)$ gives the desired result. 


\section{ElKies' METHOD FOR ATKIN PRIMES}

4.1. Computing a factor of $f_{\ell}$. Assume that $q=p$ prime, $\neq 2,3$ and that $\ell$ is an Atkin prime with $\ell \ll p$.

The $(\ell+1)$ curves $E_{i}$ are defined over $\mathbb{F}_{p^{e}}$, hence $f_{\ell}$ has a factor of degree $d$ over $\mathbb{F}_{p^{e}}$ and so by conjugation we can find a factor of degree $e d$ over $\mathbb{F}_{p}$.

First, we compute a monic irreducible factor $M_{\ell}(x)$ of degree $e$ of $\Phi_{\ell}(j(E), x)$ in $\mathbb{F}_{p}[x]$. We denote by $x_{i}, i=1,2, \ldots, e$, the roots of $M_{\ell}(x)=0$ in $\mathbb{F}_{p^{e}}$. Then, in $\mathbb{F}_{p}[x] / M_{\ell}(x)$, we determine $e d$ polynomials $p_{k}(x)=\sum_{j=0}^{e-1} a_{j, k} x^{j}$ of degree $e-1$, (see [2], [10]) and since, for $1 \leq k \leq e d$, we have

$$
p_{k} \stackrel{\text { def }}{=} \sum_{i=1}^{e} p_{k}\left(x_{i}\right)=\sum_{i=1}^{e}\left(\sum_{j=0}^{e-1} a_{j, k} x_{i}^{j}\right)=\sum_{j=0}^{e-1} a_{j, k}\left(\sum_{i=1}^{e} x_{i}^{j}\right)=\sum_{j=0}^{e-1} a_{j, k} \tilde{p}_{j}
$$

with $\tilde{p}_{j}=\sum_{i=1}^{e} x_{i}^{j}$ computed from the symmetric functions of $M_{\ell}(x)$, a factor of degree $e d$ of $f_{\ell}$ can be computed.

Example. We consider the elliptic curve $y^{2}=x^{3}+2 x+41$ over $\mathbb{F}_{59}$ with $j=31$. We determine a factor of the division polynomial $f_{5}$ of $E$. Over $\mathbb{F}_{59}, x^{3}+41 x^{2}+45 x+32$ is a factor of $\Phi_{5}(x, 31)$. We obtain

\begin{tabular}{|l||c|c||c|}
\hline \hline$p_{1}(x)$ & $56 x^{2}+31 x+41$ & $p_{4}(x)$ & $16 x^{2}+11 x+6$ \\
\hline$p_{2}(x)$ & $46 x^{2}+22 x+26$ & $p_{5}(x)$ & $51 x^{2}+41 x+17$ \\
\hline$p_{3}(x)$ & $21 x^{2}+20 x+39$ & $p_{6}(x)$ & $34 x^{2}+41 x$ \\
\hline \hline
\end{tabular}

And $p_{0}=2, p_{1}=38, p_{2}=28, p_{3}=22, p_{4}=7, p_{5}=38, p_{6}=21$, hence $x^{6}+21 x^{5}+13 x^{3}+10 x^{2}+3 x+55$ is a factor of $f_{5}$ over $\mathbb{F}_{59}$.

4.2. Computing $t \bmod \ell$. We show how, when $\ell$ is an Atkin prime, we can test the equation $\pi_{\ell}^{2}+k=\tilde{\tau} \pi_{\ell}$ in $\tilde{\tau}$ by computing only $x$-coordinates of points. We recall first that if

$$
\left(x_{1}, y_{1}\right) \oplus\left(x_{2}, y_{2}\right)=\left(x_{3}, y_{3}\right) \quad \text { and } \quad\left(x_{1}, y_{1}\right) \ominus\left(x_{2}, y_{2}\right)=\left(x_{4}, y_{4}\right),
$$

then we have

$$
\left(x_{3}+x_{4}\right)\left(x_{1}-x_{2}\right)^{2}=S\left(x_{1}, x_{2}\right) \quad \text { and } \quad x_{3} x_{4}\left(x_{1}-x_{2}\right)^{2}=P\left(x_{1}, x_{2}\right)
$$

with

$$
S\left(x_{1}, x_{2}\right)=\left(x_{1}+x_{2}\right)\left(a_{1} a_{3}+2 a_{4}+2 x_{1} x_{2}\right)+x_{1} x_{2}\left(a_{1}^{2}+4 a_{2}\right)+4 a_{6}+a_{3}^{2},
$$

and

$$
P\left(x_{1}, x_{2}\right)=\left(x_{1} x_{2}-a_{4}\right)\left(x_{1} x_{2}-a_{4}-a_{1} a_{3}\right)-\left(x_{1}+x_{2}+a_{2}\right)\left(a_{3}^{2}+4 a_{6}\right)-a_{1}^{2} a_{6} .
$$

So the values $x_{3}$ and $x_{4}$ are solutions of the quadratic equation $E(X)=N X^{2}-$ $S X+P$ with $N\left(x_{1}, x_{2}\right)=\left(x_{1}-x_{2}\right)^{2}$.

Following Müller's idea, we introduce the integers $i, j$ and $k_{o p t}$ with the equation $i \pi_{\ell}^{2}+i k=j \pi_{\ell}$. We search a value $j$ for which $x\left(j \pi_{\ell}\right)$ is a root of $E(X)=0$ given by $S\left(x_{i}^{q^{2}}, x_{i k}\right), P\left(x_{i}^{q^{2}}, x_{i k}\right)$ and $N\left(x_{i}^{q^{2}}, x_{i k}\right)$.

Indeed, if $x\left(i \pi_{\ell}^{2}+i k\right)=x\left(j \pi_{\ell}\right)$, then, for some $\tau_{0}, \pi_{\ell}^{2}+k= \pm \tau_{0} \pi_{\ell}$ over $E[\ell]$, so $\tau \equiv \pm \tau_{0} \bmod \ell$. Whereas, if $x\left(i \pi_{\ell}^{2}-i k\right)=x\left(j \pi_{\ell}\right)$, then $\pi_{\ell}^{2}-k= \pm \tau_{0} \pi_{\ell}$ and $\pi_{\ell}=2 k /\left(\tau \pm \tau_{0}\right)$, which is impossible since $\ell$ is an Atkin prime.

Hence, we avoid the computation of $y^{q^{2}}$ and $y^{q}$ and obtain $t \equiv \pm \tau_{0} \bmod \ell$. 
4.3. The sign of $t \bmod \ell$. Since $\pi_{\ell}$ satisfies the equation $x^{2}-\tau x+k=0$, we have $\pi_{\ell}^{n}=Q_{n} \pi_{\ell}+P_{n}$ with $P_{n}$ and $Q_{n}$ some polynomials in $\tau$ and $k$. We have $P_{n}=-k Q_{n-1}$ and moreover the polynomial $Q_{n}$ contains only even powers of $\tau$ if $n$ is odd and only odd powers otherwise [3]. On the other hand, $\pi_{\ell}^{e}=P_{e}$ and the value of $e$ does not depend on the sign of $\tau$. Hence, when $e$ is odd, we have $P_{e}( \pm \tilde{\tau}, k)= \pm P_{e}(\tilde{\tau}, k)$, so $\pi_{\ell}^{e}= \pm P_{e}\left(\tau_{0}, k\right)$. Let $w_{0}$ be $P_{e}\left(\tau_{0}, k\right)$.

Proposition 3. Assume $p \neq 2$, e odd; let $h_{\ell}$ be a factor of degree ed of $f_{\ell}, g_{\ell}$ be a factor of degree es $\left(w_{0}\right)$ of $h_{\ell}$ and $r$ be $\operatorname{Resultant}\left(g_{\ell}, \mathcal{G}\right)$. Then, when $s\left(w_{0}\right)$ is odd, we have $t \equiv\left(\frac{r}{q}\right) w_{0}^{s\left(w_{0}\right)} \tau_{0} \bmod \ell$. When $\ell \equiv 3 \bmod 4$, one can take $g_{\ell}=h_{\ell}$.

Proof. We have $\pi_{\ell}^{e}= \pm w_{0} I d$ over $E[\ell]$; hence, if $s\left(w_{0}\right)$ is odd, then $\pi_{\ell}^{e s\left(w_{0}\right)}=$ $\pm w_{0}^{s\left(w_{0}\right)} I d$ over $E[\ell]$ and, if $d$ is odd, then $\pi_{\ell}^{e d}= \pm w_{0}^{d} I d= \pm\left(\frac{w_{0}}{\ell}\right) I d$ over $E[\ell]$.

From $\pi_{\ell}^{2}=\tau_{0} \pi_{\ell}+k$, we easily compute $w_{0}=P_{e}\left(\tau_{0}, k\right)$. The decomposition type of $h_{\ell}$ is determined by computing $s\left(w_{0}\right)$.

Example. Let us consider the curve $y^{2}=x^{3}+4312 x+9167$ over $\mathbb{F}_{12853}$. If $\ell=19$, then we have $e=5$ and using a factor $h_{19}$ of degree 45 of $f_{19}$ we obtain $t \equiv \pm 7 \bmod 19$. We compute $r=\operatorname{Resultant}\left(x^{3}+4312 x+9167, h_{19}\right)=11226$; since $\left(\frac{r}{p}\right)=1$ and $w_{0}=P_{5}(7,9)=4$, we have $t \equiv 7 \bmod 19$.

If $\ell=13$, then $e=7$ and $\tau_{0}=5$. Since $w_{0}=P_{7}(5,9)=10$, and $s(10)=3$, the polynomial $h_{13}$ has an irreducible factor $g_{13}$ of degree 21 . We obtain $r=$ $\operatorname{Resultant}\left(x^{3}+4312 x+9167, g_{13}\right)=9515$ and $\left(\frac{r}{p}\right)=-1$, so we have $t \equiv 5 \bmod 13$.

Proposition 4. Let $h_{\ell}$ be a factor of degree ed of $f_{\ell}$. If $p=2$ and $e$ is odd, then, when $s\left(w_{0}\right)$ is odd, we have

$$
\tau=\left\{\begin{array}{cl}
w_{0}^{s\left(w_{0}\right)} \tau_{0} & \text { if } \operatorname{Tr}\left(\tilde{s}_{1}+B\left(\tilde{s}_{e s\left(w_{0}\right)-1}^{2}-2 \tilde{s}_{e s\left(w_{0}\right)} \tilde{s}_{e s\left(w_{0}\right)-2}\right) / \tilde{s}_{e s\left(w_{0}\right)}^{2}\right)=0, \\
-w_{0}^{s\left(w_{0}\right)} \tau_{0} & \text { otherwise, }
\end{array}\right.
$$

with $\tilde{s}_{i}$ the symmetric functions of a factor $g_{\ell}$ of $h_{\ell}$ of degree es $(w)$. When $\ell \equiv$ $3 \bmod 4$, one can take $g_{\ell}=h_{\ell}$.

\section{ACKNOWLEDGMENT}

The author would like to thank François Morain for his help during the realization of this work.

\section{REFERENCES}

[1] A. O. L. Atkin, The number of points on an elliptic curve modulo a prime (I). Draft, 1988.

[2] A. O. L. Atkin, The number of points on an elliptic curve modulo a prime (II). Draft, 1992.

[3] R. C. Bose, S. Chowla, C. R. Rao, On the integral order ( $\bmod p)$ of quadratics $x^{2}+a x+b$, with applications to the construction of minimum functions over $G F\left(p^{2}\right)$, and to some number theory results, Bull. Calcutta Math. Soc., 36 (1944), pp. 153-174. MR 6:256b

[4] R. P. Brent, H. T. Kung, Fast algorithms for manipulating formal power series, J. Assoc. Comput. Mach., 25, 581-595, 1978. MR 58:25090

[5] J.-M. Couveignes, F. Morain, Schoof's algorithm and isogeny cycles, In L. Adleman and M.-D. Huang, editors, ANTS-I, volume 877 of Lecture Notes in Comput. Sci., pages 43-58. Springer-Verlag, 1994. MR 95m:11147

[6] J.-M. Couveignes, L. Dewaghe, F. Morain, Isogeny cycles and Schoof's algorithm, Preprint 1995.

[7] J.-M. Couveignes, Quelques calculs en théorie des nombres, Thèse, Université de Bordeaux I, July 1994. 
[8] L. Dewaghe, Nombre de points d'une courbe elliptique sur un corps fini, Thèse, Université de Lille I, 1996.

[9] R. J. McEliece, Finite fields for computer scientists and engineers, Kluwer Boston 1987. MR 88h:11091

[10] N. D. Elkies, Explicit Isogenies, Draft, 1991.

[11] D. E. Knuth, The Art of Computer Programming : Seminumerical Algorithms, AddisonWesley, 1981. MR 83i:68003

[12] R. Lercier and F. Morain, Counting the number of points on elliptic curves over finite fields: strategies and performances, In L. C. Guillou and J.-J. Quisquater, editors, Advances in cryptology - EUROCRYPT'95, number 921 of Lecture Notes in Comput. Sci. pp. 79-94, 1995. MR 96h: 11060

[13] R. Lercier, F. Morain, Counting points on elliptic curves over $\mathbb{F}_{p^{n}}$ using Couveignes's algorithm. Research Report LIX/RR/95/09, Ecole polytechnique - LIX, September 1995.

[14] A. Menezes, S. Vanstone and R. Zuccherato, Counting points on elliptic curves over $\mathbb{F}_{2^{m}}$, Math. Comp. 60, 407-420, 1993. MR 93f:11098

[15] F. Morain, Calcul du nombre de points sur une courbe elliptique dans un corps fini : aspects algorithmiques, J. Théor. Nombres Bordeaux 7 (1995), 255-282. MR 97i:11069

[16] V. Müller, Looking for the eigenvalue in Schoof's algorithm, In preparation, October 1994.

[17] R. Schoof, Elliptic curves over finite fields and the computation of square roots mod $p$, Math. Comp. 44, 483-494, 1985. MR 86e: 11122

[18] R. Schoof, Counting points on elliptic curves over finite fields, J. Théor. Nombres Bordeaux 7 (1995), 219-254. MR 97i: 11070

[19] V. Shoup, A new polynomial factorization algorithm and its implementation, J Symbolic Comput. (1995) Vol 20, 363-397. MR 97d:12011

[20] J. H. Silverman, The arithmetic of elliptic curves, vol. 106 of Graduate Texts in Mathematics, Springer, 1986. MR 87g:11070

Université de Lille I, UFR de Mathématiques, 59655 Villeneuve d'AscQ Cedex, France E-mail address: dewaghe@gat.univ-lille1.fr 